\title{
CLASSIFICATION OF INTRAVASCULAR ULTRASOUND IMAGES BASED ON NON-NEGATIVE MATRIX FACTORIZATION FEATURES AND MAXIMUM LIKELIHOOD CLASSIFIER
}

\author{
Dr. Rajan A, \\ Department of Electronics and Communication Engineering, \\ Geethanjali Institute of Science and Technology, \\ Nellore, Andhra Pradesh, India \\ arurajan79@gmail.com
}

\begin{abstract}
The amount of plaque in coronary arteries in any particular point is identified by the IntraVascular UltraSound (IVUS) images. The classification of IVUS images is very important to diagnose various coronary artery diseases. In this study, the classification of IVUS images based on Non-negative Matrix Factorization (NMF) technique and Maximum Likelihood Classifier (MLC) is presented. Initially, the IVUS images are given to frost filter to remove speckle noise as the imaging technique uses ultrasound waves. Then, NMF technique is employed to extract the features and stored in database. Then MLC is used for classification of IVUS images for both normal and abnormal categories. The IVUS Image Classification (IIC) system obtains $98 \%$ classification accuracy by using NMF features and MLC classification.
\end{abstract}

Keywords: Intravascular ultrasound image classification, frost filtering, nonnegative matrix factorization, maximum likelihood classifier.

\section{INTRODUCTION}

IVUS images are mainly used for the diagnosis of coronary artery disease. Also, the progression and regressed lesions in the arteries are identified by using IVUS images. IVUS images based blood region classification using three dimensional brushlet expansions is described in [1]. The input IVUS images are given to brushlet analysis. Features like combining image information and geometric constraints are extracted. Neural network classifier is used for classification. Characterization of IVUS images based on feature selection and Support Vector Machine (SVM) classification is presented in [2]. Initially, the borders of the IVUS image are detected. Features like first-order statistics, Local Binary Pattern (LBP), Gray-level Co-Occurrence Matrix (GLCM), run length features and wavelet features are extracted.

Coronary plaque classification using IVUS image is discussed in [3]. Texture features like LBP, Gabor filters and co-occurrence matrix are extracted. Adaptive boosting classifier is used for classification. Classification and differentiation of lesions in the IVUS images are discussed in [4]. At first, the two and three dimensional texture features are extracted. Principal component analysis is used to reduce the features. Classification is made by three and two dimensional classifier.

Plaque classification from IVUS images is discussed in [5]. The input IVUS images are given to co-occurrence matrix, fractal measure, GLCM and run length modules to extract features. The hard plaque regions are classified. IIC system by genetic fuzzy rule based approach is described in [6]. Features like GLCM, first 
order statistics, LBP, run length and local indicators of spatial association are extracted. Classification is made by fast iterative rule-based linguistic classifier.

Textural approach for the detection of soft and hard plaque is discussed in [7]. Features like texture and GLCM are extracted. The detection of soft and hard plaque is made by fuzzy clustering means algorithm. Analysis of plaque tissues using IVUS images is discussed in [8]. Feature extraction is made by the combination of texture with statistical features and then classification is made by fisher linear discriminant analysis.

IVUS image filtering using wavelet and non local means filter is discussed in [9]. Initially the IVUS images are given to wavelet transform that produce approximate and detailed coefficients. The approximation coefficient is given to non local means filter. The inverse transform is performed by detailed coefficient and non local means filter. Shadow detection in IVUS images using adaptive thresholding is discussed in [10]. At first, the IVUS images are preprocessed by circle Hough histogram to remove catheter zone. Otsu thresholding and active contour methods are used for segmentation.

Characterization of IVUS image is described in [11]. The region of interest area is extracted from the IVUS images. Then, the borders are detected in the current frame by active contour method. Features are extracted by GLCM, fractal based measures and run length. Then the soft and hard plaques are characterized. A classification system for IVUS images is discussed in [12]. At first, texture features are extracted. The state of art classifiers like SVM, adaptive boosting a random forest classifier is used for classification.

In this paper, NMF features based IIC system is presented. The organization of paper is as follows: The methods and materials used for IIC are described in section 2. In section 3 results and discussion of IIC are presented. Final section concludes the IIC system by NMF features and MLC classification.

\section{METHODS AND MATERIALS}

The IIC system using NMF features and MLC is shown in Fig 1. It uses preprocessing, feature extraction and classification stages to classify the IVUS images. These three stages are explained in the following sub-sections.

\section{A. Speckle de-noising}

An IVUS image contains speckle noise due to the use of transducer which produces echo waves. Speckle noises are produced due to air gap between the transducer and body. It reduces the resolution and contrast of the image that could reduce the diagnostic value. Thus, the removal of speckle noise is essential. In this study, frost filter is used for de-noising of IVUS images. It is also used in multilayer histogram equalization [13] and anisotropic diffusion [14]. The frost filter works in the edges to remove noise. The key factor known as damping factor controls the filters smoothness. The image is smooth with the small damping factor. The frost filter is given by,

$$
Z=\frac{\sum(y * U)}{\sum U}
$$

where $y$ is the pixel value, $Z$ is a filtered pixel value and $U$ is a weight for each pixel. 


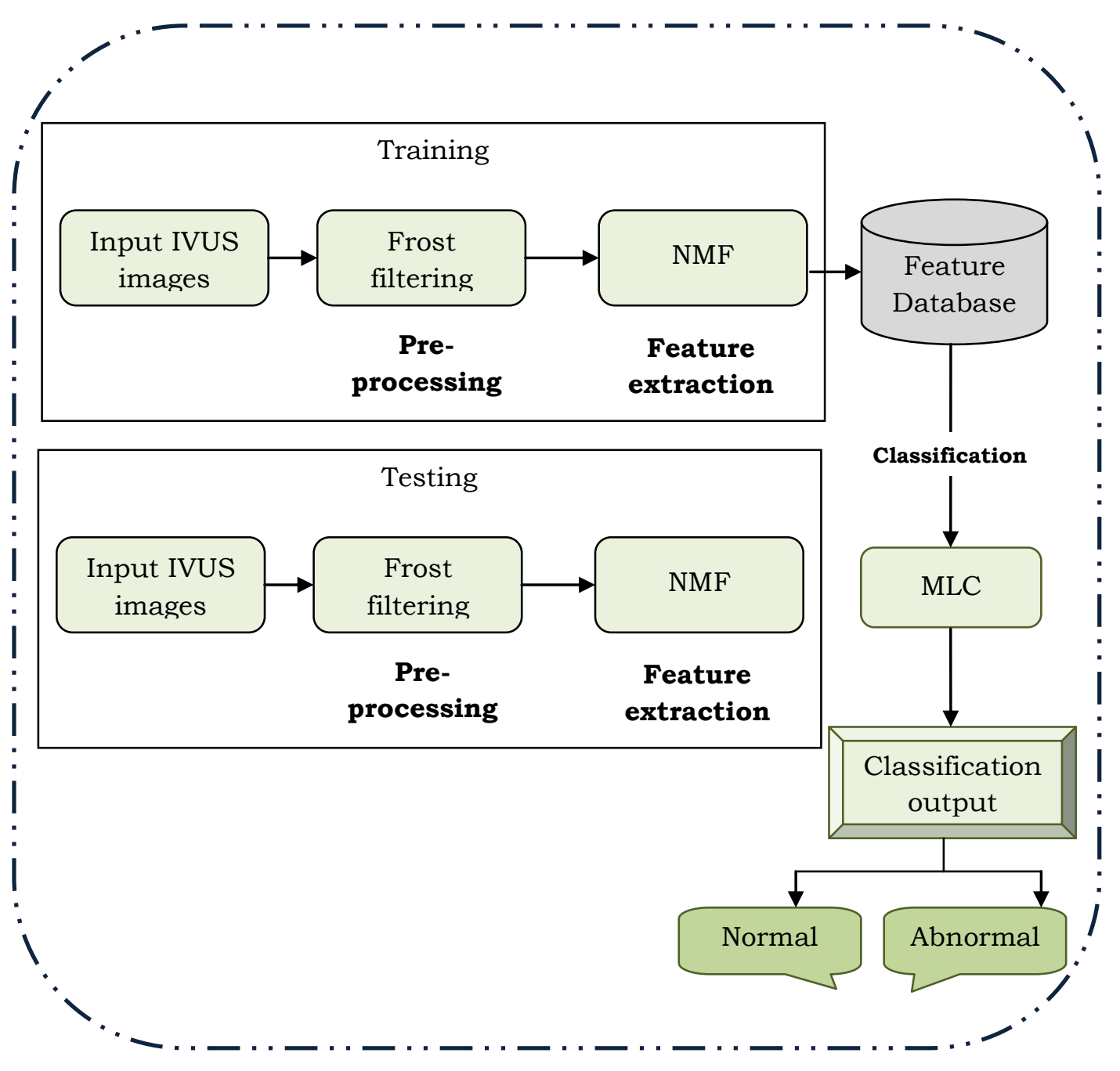

Fig. 1 IIC system using NMF features and MLC classification

\section{B. NMF Features}

$\mathrm{NMF}$ is a group of algorithm in linear algebra and multivariate analysis. Figure 2 shows the architecture of NMF method.

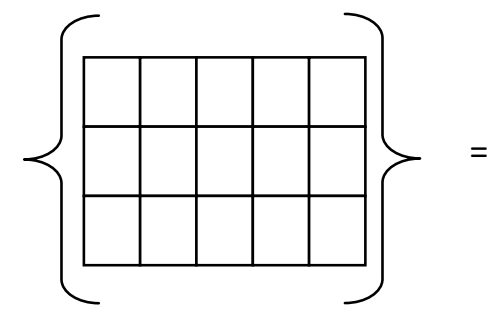

Matrix $K$

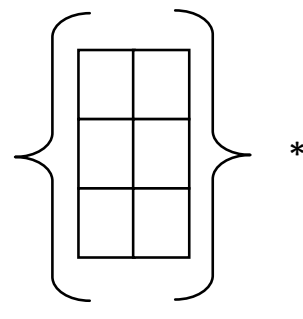

$\mathrm{L}$

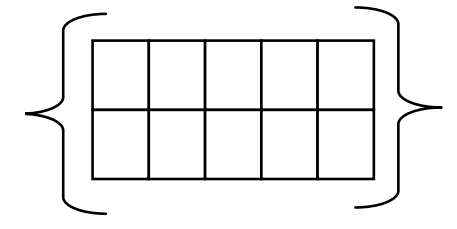

M

Fig. 2 NMF method for factorization 
For example, a matrix $K$ is factorized into two matrices $L$ and $M$, with the property that all three matrices have no negative elements. It is easy to check the result matrices. Let us consider the matrix $K$, be the product of matrices $L$ and $M$. where $K=L M$, The factorization is made by the column vectors of $K$ as linear combinations of column vector in $L$ using coefficients supplied by columns of $M$. The column vector $K$ is evaluated by $k_{i}=L m_{i}$, where $k_{i}$ is the $i^{\text {th }}$ column vector of the product matrix $K$ and $m_{i}$ is the $i^{\text {th }}$ column vector of the matrix $K$. NMF is also used as the probabilistic graphical model which is shown in Fig. 3.

This model shows the visible units $(K)$ which is connected to all hidden units $(M)$ by the weight $(L)$, so that the $K$ is generated to the probability distribution function and it is defined by,

$$
\sum_{\gamma} M_{i \gamma} L_{\gamma}
$$

where $M_{i}$ is the hidden unit, $L$ is the weight of the hidden unit. NMF is also used in glass defect identification [15] and channel source separation [16]. In this study, NMF is used for feature extraction of preprocessed image. It extracts the image by visible and hidden units for both testing and training and saved in the feature database.

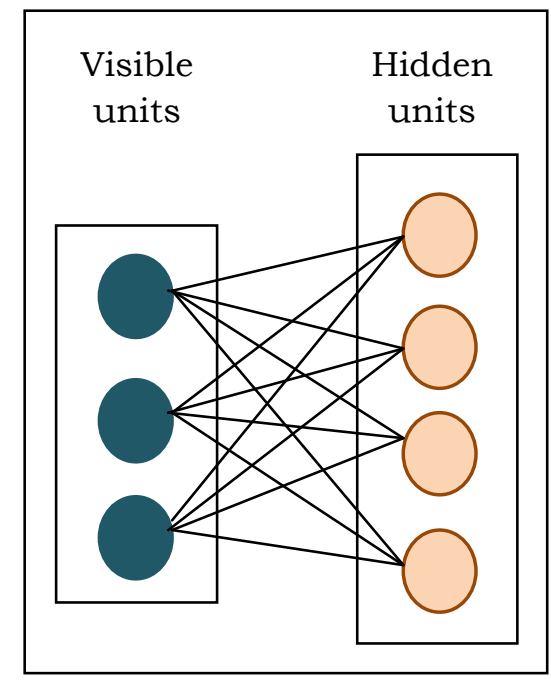

Fig. 3 Probabilistic graphical model used in NMF

\section{MLC Classification}

MLC is used in the classification of remote sensing with the maximum likelihood pixel value and classified into the correct class. It is also used in other fields like texture classification [17] and tunneled traffic [18]. If all pixels are classified in the probability threshold then each pixels are assigned to the class with highest probability. The threshold is greater than the highest probability then the pixels are not classified. The likelihood $(L h)$ which belongs to the class $v$ is defined by,

$$
L h=H(v / Y)=H(v) * H(Y / v) / \sum H(i) * H(Y / i)
$$

where $H(v)$ is the probability of class $v . P(Y / v)$ is the probability to observe $Y$ from class $v$, otherwise it is known as probability density function. 


\section{RESULTS AND DISCUSSION}

The performance evaluation of IIC system using 100 IVUS images (50 normal and 50 abnormal) is described in this section. The performance of IIC system is measured by classification accuracy. Figure 4 shows the sample normal and abnormal images in IVUS dataset.

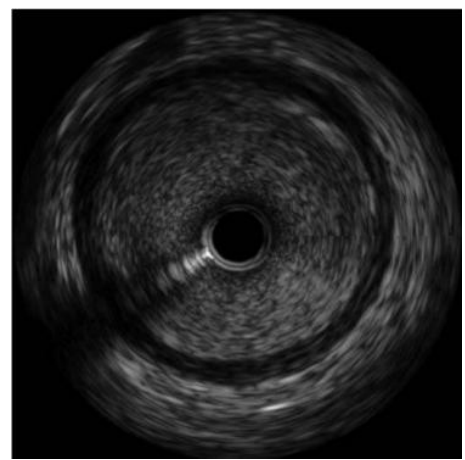

(a)

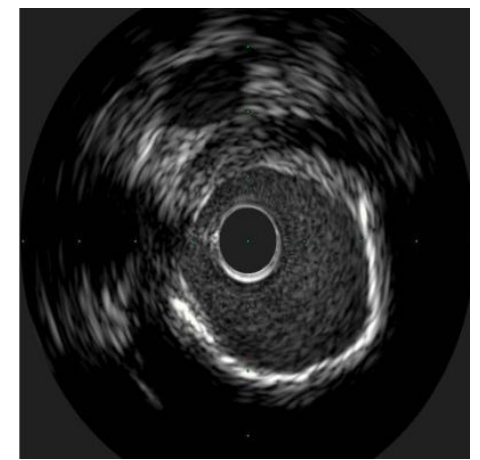

(b)

Fig. 4 (a) Normal IVUS image (b) Abnormal IVUS image

The input normal and abnormal IVUS images are de-noised by frost filter to remove speckle noise at preprocessing stage. The noise removed images are processed to extract NMF features and it has the key hypermeter which is set to $S$ $=0.7 n$ for better performance evaluation. Both $S$ and $n$ reduce the features and it also increases the classification accuracy. Then these features are stored in feature database. MLC is used for classification. Table 1 shows the classification accuracy of IIC system using NMF and MLC.

TABLE 1 Classification accuracies of IIC system using NMF and MLC

\begin{tabular}{|c|c|c|}
\hline $\mathbf{S}$ & $\begin{array}{c}\text { NMF Computation time } \\
\text { (seconds) }\end{array}$ & $\begin{array}{c}\text { Classification accuracy } \\
\text { (\%) }\end{array}$ \\
\hline 17 & 5.16 & 79.00 \\
\hline 31 & 8.03 & 85.00 \\
\hline 45 & 5.06 & 93.50 \\
\hline 59 & 5.37 & 96.00 \\
\hline 73 & 5.09 & 98.00 \\
\hline 87 & 5.29 & 97.50 \\
\hline 101 & 5.27 & 96.00 \\
\hline 115 & 5.44 & 92.50 \\
\hline 129 & 5.65 & 90.00 \\
\hline 143 & 13.25 & 84.50 \\
\hline
\end{tabular}

It is observed from above table the classification accuracy increases as the hypermeter value increases up to 73 . The highest classification accuracy of $98 \%$ is obtained at key hypermeter value of 73 . It takes 5.27 seconds to make a decision. The minimum classification accuracy of $79 \%$ is obtained at key hypermeter value of 17 by MLC for IIC system. 


\section{CONCLUSION}

An efficient method for IIC system for plaque identification in coronary artery using NMF and MLC using IVUS images is presented. The IVUS images have the speckle noise due to loss of proper contact. The input IVUS images are preprocessed to remove speckle noise using frost filter. Then the preprocessed images are given to NMF for feature extraction. It uses key parameter $S$ and the extracted NMF feature values are saved in the feature database and used for classification. MLC is used to classify IVUS images into normal or abnormal. A better classification accuracy of $98 \%$ is obtained by using NMF with 73 as a key parameter values and MLC.

\section{REFERENCES}

[1]. A. Katouzian, M.A. Selver, E.D. Angelini, B. Sturm, and A.F. Laine, "Classification of blood regions in IVUS images using three dimensional brushlet expansions", International Conference of the IEEE Engineering in Medicine and Biology Society, 2009, pp. 471-474.

[2]. V.G. Giannoglou, D.G. Stavrakoudis, and J.B. Theocharis, "IVUS-based characterization of atherosclerotic plaques using feature selection and SVM classification", International Conference on Bioinformatics \& Bioengineering, 2012, pp. 715-720.

[3]. K.L. Caballero, J. Barajas, O. Pujol, O. Rodriguez, and P. Radeva, "Using reconstructed ivus images for coronary plaque classification", International Conference of the IEEE Engineering in Medicine and Biology Society, 2009, pp. 2167-2170.

[4]. P. Brathwaite, A. Nagaraj, B. Kane, D.D. McPherson, and E.L. Dove, "Automatic classification and differentiation of atherosclerotic lesions in swine using IVUS and texture features", Computers in Cardiology, 2002, pp. 109-112.

[5]. X. Zhang, S.C. DeJong, C.R. McKay, S.M. Collins, and M. Sonka, "Automated characterization of plaque composition from intravascular ultrasound images", Computers in Cardiology, 1996, pp. 649-652.

[6]. V.G. Giannoglou, D.G. Stavrakoudis, J.B. Theocharis, and V. Petridis, "Genetic fuzzy rule-based classification systems for tissue characterization of intravascular ultrasound images", International Conference on Fuzzy Systems, 2012, pp. 1-8.

[7]. S. Dehnavi, M.P. Babu, M. Yazchi, and M. Basij, "Automatic soft and hard plaque detection in IVUS images: A textural approach", Information \& Communication Technologies, 2013, pp. 214-219.

[8]. D. Haiyan, and L. Hong, "Texture analysis and characteristic identification about plaque tissues of IVUS", International Conference on Computational and Information Sciences, 2010, pp. 873-876.

[9]. D. China, P. Mitra, C. Chakraborty, and K.M. Mandana, "Wavelet based non local means filter for despeckling of intravascular ultrasound image", International Conference on Advances in Computing, Communications and Informatics, 2015, pp. 1361-1365.

[10]. M. Basij, P. Moallem, M. Yazdchi, and S. Mohammadi, "Automatic shadow detection in intra vascular ultrasound images using adaptive thresholding", International Conference on Systems, Man, and Cybernetics, 2012, pp. 2173-2177. 
[11]. X. Zhang, C.R. McKay, and M. Sonka, "Tissue characterization in intravascular ultrasound images", IEEE Transactions on Medical Imaging, Vol.17, No.6, 1998, pp. 889-899.

[12]. M. Alberti, S. Balocco, C. Gatta, F. Ciompi, O. Pujol, J. Silva, X. Carrillo, and P. Radeva, "Automatic bifurcation detection in coronary IVUS sequences", IEEE transactions on biomedical engineering, Vol.59, No.4, 2012, pp.1022-1031.

[13]. C.S. Josephus, and S. Remya, "Multilayered contrast limited adaptive histogram equalization using frost filter", IEEE Recent Advances in Intelligent Computational Systems, 2011, pp. 638-641.

[14]. G. Chen, X. LIVUS, and Z. Zhou, "Modified frost speckle filter based on anisotropic diffusion", IET International Conference on Radar Systems, 2007, pp. 1-4.

[15]. Y. Bao, Z. Qibing, and H. Min, "Image identification of glass defects based on Non-Negative Matrix Factorization and Sparse Representation Classification", Chinese Control and Decision Conference, 2012, pp. 32253229.

[16]. B. Gao, W.L. Woo, and S.S. Dlay, "Adaptive sparsity non-negative matrix factorization for single-channel source separation", IEEE journal of selected topics in signal processing, Vol. 5, No. 5, 2011, 989-1001.

[17]. K.S. Thyagarajan, T. Nguyen, and C.E. Persons, "A maximum likelihood approach to texture classification using wavelet transform", International Conference on Image Processing, 1994, pp. 640-644.

[18]. X. Tan, X. Su, and Q. Qian, "The classification of SSH tunneled traffic using maximum likelihood classifier", International Conference on Electronics, Communications and Control, 2011, pp. 2347-2350. 